\title{
Controle da Pressão Arterial: O Segredo é... Trabalho em Equipe!
}

\author{
Blood Pressure Control: The secret is...Team Work!
}

\author{
Andrea Pio-Abreu ${ }^{1}$ e Luciano F. Drager ${ }^{1,2}$ \\ Unidade de Hipertensão, Divisão de Nefrologia, Faculdade de Medicina da Universidade de São Paulo, ${ }^{1}$ São Paulo, SP - Brasil \\ Unidade de Hipertensão, Instituto do Coração (InCor), Faculdade de Medicina da Universidade de São Paulo, ${ }^{2}$ São Paulo, SP - Brasil \\ Minieditorial referente ao artigo: Controle da Pressão Arterial e Fatores Associados em um Serviço Multidisciplinar de Tratamento da Hipertensão
}

A hipertensão é uma das principais causas de morte cardiovascular. ${ }^{1}$ De fato, dados do Heart and Stroke Statistics mostraram que $45 \%$ da mortalidade cardiovascular é potencialmente atribuída à hipertensão. ${ }^{1}$ Esse cenário preocupante não mudou nas últimas décadas, apesar da disponibilidade das intervenções não-farmacológicas e o desenvolvimento de várias classes de remédios antihipertensivos que efetivamente contribuíram para o controle da pressão arterial (PA). ${ }^{2-5}$ As razões de nossa baixa efetividade no controle da PA no nível populacional são múltiplas, incluindo a falta de políticas públicas organizadas que regulem o consumo de sal e aumentem a conscientização, a detecção precoce e o tratamento efetivo. Desafios adicionais incluem a característica assintomática da hipertensão, inércia terapêutica, entre outros. ${ }^{1}$

Nesta edição dos Arquivos Brasileiros de Cardiologia, Jardim et al., ${ }^{6}$ relataram dados de um estudo retrospectivo explorando uma estratégia de equipe multidisciplinar na taxa de controle da pressão arterial (estabelecida no tradicional $<140 / 90 \mathrm{mmHg}$ ). Os autores avaliaram dados demográficos e clínicos de 1.548 pacientes hipertensos de um centro especializado em hipertensão, acompanhados regularmente por 7,6 \pm 7,1 anos (média de idade de 62 anos, 73,6\% de mulheres).

A abordagem multidisciplinar descrita pelos autores consistiu na disponibilidade de enfermeiros, nutricionistas, terapeutas ocupacionais, educadores físicos, psicólogos e musicoterapeutas trabalhando em conjunto com os médicos da equipe (clínicos gerais, cardiologistas, endocrinologistas e nefrologistas). O intervalo máximo para consultas médicas foi de 3 meses. De acordo com as necessidades dos pacientes (determinados pela avaliação clínica), os médicos agendavam visitas aos profissionais mencionados acima em uma demanda flexível. Além disso, foram realizadas atividades educacionais e de promoção da saúde a cada duas semanas com os pacientes. Toda essa informação foi registrada em um

\section{Palavras-chave}

Hipertensão; Pressão Arterial; Prevenção e Controle; Fatores de Risco; Equipe de Assistência ao Paciente/ tendências; Anti-Hipertensivos; Adesão à Medicação.

Correspondência: Luciano F. Drager •

Unidade de Hipertensão, Instituto do Coração (InCor), Faculdade de Medicina da Universidade de São Paulo - Av. Dr. Eneas de Carvalho Aguiar, 44. CEP 05403-900, São Paulo, SP - Brasil.

E-mail: luciano.drager@incor.usp.br

DOI: https://doi.org/10.36660/abc.20200544 formulário padronizado. Usando essa estratégia, os autores descobriram que essa abordagem de equipe multidisciplinar estava associada a um controle geral da pressão arterial de $68 \%$, sendo mais acentuado naqueles com idade $\geq 60$ anos (OR 1,45; IC 95\% [1,13-1,90]) e em mulheres (OR 1,36; IC95\% [1,09-1,88]). Por outro lado, pacientes com diabetes foram associados a uma menor probabilidade de atingir a meta de PA em comparação com pacientes sem diabetes. Curiosamente, não foram observadas diferenças significativas no número de medicamentos anti-hipertensivos nos grupos que controlaram ou não a PA. Esse achado sugere que a adesão a essa abordagem multidisciplinar pode variar, como geralmente observado em qualquer outra intervenção.

Vale ressaltar o mérito do serviço relacionado, cuja prática multidisciplinar é adotada, de acordo com os autores, há mais de 25 anos. ${ }^{6} \mathrm{O}$ controle da PA é impressionante, considerando as estimativas atuais do controle da PA no Brasil (geralmente abaixo de $30 \%$ em estudos individuais). ${ }^{3}$ De maneira geral, a principal contribuição dessa investigação é que a literatura é relativamente escassa (principalmente de grandes estudos multicêntricos observacionais ou randomizados) ao abordar o impacto potencial de uma equipe multidisciplinar em pacientes com hipertensão. Estudos anteriores envolvendo tamanhos de amostras modestos sugeriram a importância dos enfermeiros na melhora da adesão a tratamentos antihipertensivos e do efeito de jaleco branco. ${ }^{6-11}$ Da mesma forma, a abordagem ativa de farmacêuticos, educadores físicos e nutricionistas parece contribuir para melhorar a adesão e o controle da PA. ${ }^{12,13}$ Entretanto, é crucial definir se toda a estrutura de equipe disponível (e não os 'compartimentos' distintos) pode contribuir para a eficácia do controle da PA. Em outras palavras, o todo é melhor do que qualquer componente individual ou a soma das partes?

O estudo realizado por Jardim et al. não foi projetado para abordar essa questão, mas destacou que a luta contra a hipertensão não se baseia em um único ator. Infelizmente, a falta de um grupo controle (por exemplo, pacientes de outros centros sem acesso a uma abordagem de equipe multidisciplinar organizada) e o desenho retrospectivo impedem conclusões definitivas, mas abrem caminho para futuras investigações nesta importante área de pesquisa. Atenção especial deve ser dedicada aos pacientes com diabetes. A menor taxa de controle da PA nos desafia a realizar esforços extras nessa população de alto risco cardiovascular. 


\section{Referências}

1. Virani SS, Alonso A, Benjamin EJ, Bittencourt MS, Callaway CW, Carson AP, et al. Heart Disease and Stroke Statistics-2020 Update: A Report From the American Heart Association. Circulation. 2020;141(9):e139-e596.

2. Sever PS, Messerli FH. Hypertension management 2011: optimal combination therapy. Eur Heart J. 2011;32(20):2499-506.

3. Malachias MVB, Póvoa RMS, Nogueira AR, Souza D, Costa LS, Magalhães ME. 7a Diretriz Brasileira de Hipertensão Arterial: Capítulo 3 - Avaliação Clínica e Complementar. Arq Bras Cardiol. 2016;107(3 Supl.3):1-83.

4. Krieger EM, Drager LF, Giorgi DMA, Pereira AC, Barreto-Filho JAS, Nogueira AR, et al. Spironolactone versus clonidine as a fourthdrug therapy for resistant hypertension: The ReHOT randomized study (Resistant Hypertension Optimal Treatment). Hypertension. 2018;71(4):681-90.

5. Pio-Abreu A, Drager LF. Resistant hypertension: time to consider the best fifth anti-hypertensive treatment. Curr Hypertens Rep. 2018;20(8):67.

6. Basilio PG, Oliveira APC, Castro ACF, Carvalho MB, Zagatto AM, Martinez PF, et al. Intermittent Fasting Attenuates Exercise TrainingInduced Cardiac Remodeling. Arq Bras Cardiol. 2020; 115(2):184-193.
7. Barkauskas VH, Pohl JM, Tanner C, Onifade TJ, Pilon B. Quality of care in nurse-managed health centers. Nurs Adm Q. 2011;35(1):34-43.

8. Himmelfarb CR, Commodore-Mensah Y, Hill MN. Expanding the role of nurses to improve hypertension care and control globally. Ann Glob Health. 2016;82(2):243-53.

9. Rudd P, Miller NH, Kaufman J, Kraemer HC, Bandura A, Greenwald G, et al Nurse management for hypertension. A systems approach. Am J Hypertens. 2004;17(10):921-7.

10. Carter BL, Bosworth HB, Green BB. The hypertension team: the role of the pharmacist, nurse, and teamwork in hypertension therapy. J Clin Hypertens (Greenwich). 2012;14(1):51-65.

11. Guerra-Riccio GM, Giorgi DMA, Consolin-Colombo FM, Barreto-Filho JAS, Lopes HF, Camargo ALF, et al. Frequent nurse visits decrease white coat effect in stage III hypertension. Am J Hypertens. 2004;17(6):523-8.

12. Borenstein JE, Graber G, Saltiel E, Wallace J, Ryu S, Archi J, et al. Physicianpharmacist comanagement of hypertension: a randomized, comparative trial. Pharmacotherapy. 2003;23(2):209-16.

13. Han E, Sohn HS, Lee JY, Jang S. Health behaviors and medication adherence in elderly patients. Am J Health Promot. 2017;31(4):278-86. 